
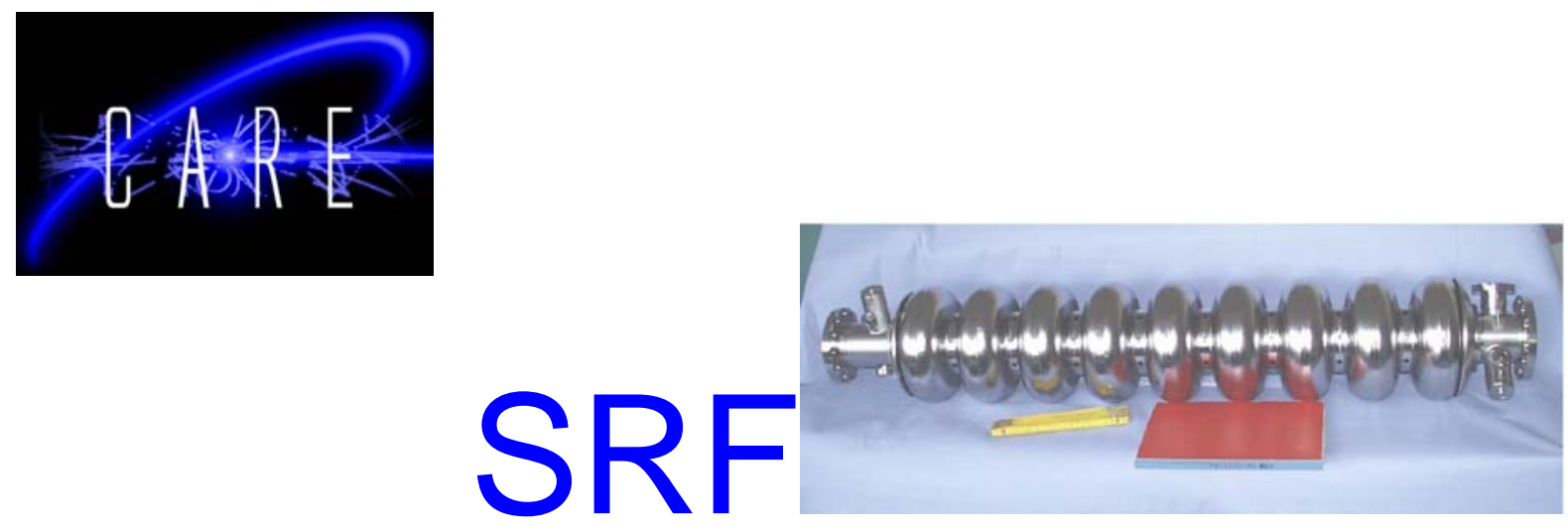

\title{
Characterization at Cryogenic Temperatures of Piezostacks Dedicated to Fast Tuners for SRF Cavities
}

M. Fouaidy, G. Martinet, N. Hammoudi, F. Chatelet, A. Olivier

IPN Orsay, France

\begin{abstract}
In the frame of the European CARE project, we designed and constructed three apparatus dedicated to full characterization of low voltage piezoelectric actuators. These piezostacks are integrated in Fast Active Cold Tuning Systems (FACTS) and used for dynamic stabilisation of the resonant frequency of Superconducting RF (SRF) cavities. The three facilities we developed allow the measurement of electromechanical (capacitance, loss factor, displacement), thermal (specific heat, interfacial thermal resistance) and dynamic properties (effect of a preloading force) of industrial piezostacks for temperature in the range $2 \mathrm{~K}-300 \mathrm{~K}$. Moreover, several piezostacks supplied by three different companies were subjected to ionizing radiation with fast neutrons beams at $\mathrm{T}=4.2 \mathrm{~K}$ and for fluence in excess of $2.1014 \mathrm{n} / \mathrm{cm} 2$. The test apparatus are described in details then the experimental data are analyzed and discussed.
\end{abstract}

Contribution to the "MIXDES 2007" Conference, Ciechocinek (Poland), 21-23 June 2007

Work supported by the European Community-Research Infrastructure Activity under the FP6 "Structuring the European Research Area" programme (CARE, contract number RII3-CT2003-506395) 


\title{
CHARACTERIZATION AT CRYOGENIC TEMPERATURES OF PIEZOSTACKS DEDICATED TO FAST TUNERS FOR SRF CAVITIES
}

\author{
M. Fouaidy, G. Martinet, N. Hammoudi, F. Chatelet, A. Olivier \\ IPNO*, FRANCE
}

\section{KEYWORDS: Superconducting RF cavities, Fast tuning, Piezoelectric actuator, Low temperature}

\begin{abstract}
In the frame of the European CARE project, we designed and constructed three apparatus dedicated to full characterization of low voltage piezoelectric actuators. These piezostacks are integrated in Fast Active Cold Tuning Systems (FACTS) and used for dynamic stabilisation of the resonant frequency of Superconducting RF (SRF) cavities. The three facilities we developed allow the measurement of electromechanical (capacitance, loss factor, displacement), thermal (specific heat, interfacial thermal resistance) and dynamic properties (effect of a preloading force) of industrial piezostacks for temperature in the range $2 \mathrm{~K}-300 \mathrm{~K}$. Moreover, several piezostacks supplied by three different companies were subjected to ionizing radiation with fast neutrons beams at $\mathrm{T}=4.2 \mathrm{~K}$ and for fluence in excess of $2.10^{14} \mathrm{n} / \mathrm{cm}^{2}$. The test apparatus are described in details then the experimental data are analyzed and discussed.
\end{abstract}

\section{SRF CAVITIES AND LORENTZ DETUNING}

Superconducting RF (SRF) bulk niobium cavities are nowdays widely used as accelerating structures and at large scale in recent particles accelerators such as Continous Electrons Beam Accelerator Facility [1], Spallation Neutrons Source [2] and future machines either in construction such as XFEL [3] or still in R\&D phase such as International Linear Collider (ILC [4]). The choice of SRF cavities instead of normal conducting resonators is mainly dictated by accelerating efficiency considerations and RF performance: 1) thanks to superconducting properties Joule RF losses in SRF cavities are negligibly small ( $\sim$ tenths of $\mathrm{W}$ ) resulting in higher unloaded quality factor $Q_{0}$ by 5 order of magnitude in comparison to normal conducting structures, 2) as consequence, higher accelerating gradient $\mathrm{E}_{\mathrm{acc}}$ for machine duty cycles in excess of $10 \%$ are allowed. However, due to their high unloaded quality factor $\mathrm{Q}_{0}$ (e.g., $\mathrm{Q}_{0}>10^{10}$ ) and consequently a narrow bandwidth $\Delta \mathrm{f}_{\mathrm{BW}}\left(\Delta \mathrm{f}_{\mathrm{BW}} \alpha \mathrm{l} / \mathrm{Q}\right)$, SRF cavities are very sensitive to small mechanical perturbations which change the volume of the resonator. More precisely, high surface electromagnetic fields (e.g., radiation pressure) induce mechanical deformations $(\sim \mu \mathrm{m})$ of the thin $(\sim 3 \mathrm{~mm}-4 \mathrm{~mm})$ cavity wall, resulting in a frequency shift or Lorentz detuning $\Delta \mathrm{f}_{\mathrm{L}} \approx \Delta \mathrm{f}_{\mathrm{BW}}$ of these accelerating structures. Moreover, the radiation pressure $\mathrm{P}$ depends quadratically on the surface RF electric $\left(\mathrm{E}_{\mathrm{S}}\right)$ and magnetic $\left(\mathrm{H}_{\mathrm{S}}\right)$ fields according to the relationship:

$$
P=\frac{1}{4} \cdot\left(\mu_{0} H_{S}^{2}-\varepsilon_{0} E_{S}^{2}\right)
$$

As illustration, we present in Fig. 1-Fig. 3 a photograph of a $704 \mathrm{MHz}$ SRF cavity prototype developed by IPNO for high intensity protons linac, the electric field pattern in the TM010 mode [5] and the radiation pressure profile along the cavity axis.

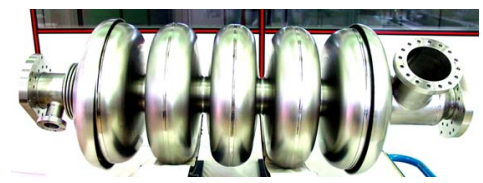

Fig. 1: SRF bulk niobium cavity (Frequency: $704 \mathrm{MHz}$, Active length $=692 \mathrm{~mm}$, Thickness $=4 \mathrm{~mm}$ )

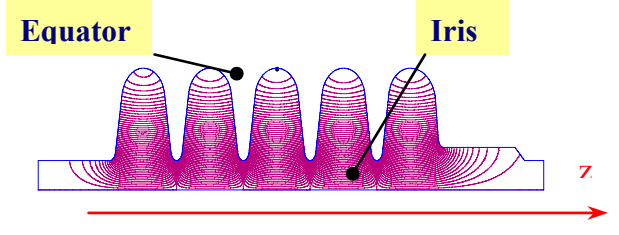

Fig. 2: Computed electric field in TM010 mode

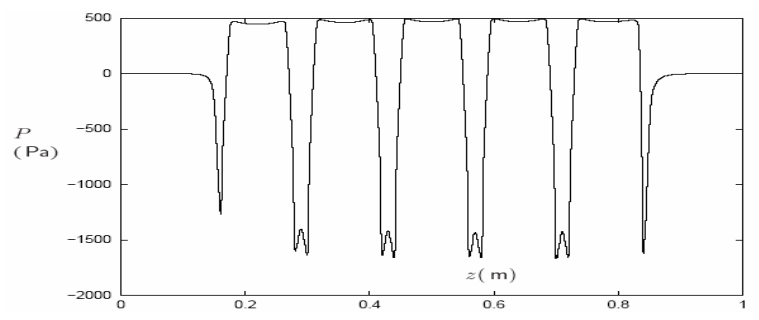

Fig. 3: Profile of the radiation pressure along the cavity axis for an accelerating field $E_{a c c}=10 \mathrm{MV} / \mathrm{m}$

In the above SRF cavity, the computed maximum displacements in axial ( $\mathrm{z}$ ) and radial directions, induced by radiation pressure when the resonator is operated at $\mathrm{E}_{\mathrm{acc}}=10 \mathrm{MV} / \mathrm{m}$ are respectively $0.32 \mu \mathrm{m}$ and $0.037 \mu \mathrm{m}$. Such deformation corresponds to a Lorentz detuning $\Delta \mathrm{f}_{\mathrm{L}}=-419 \mathrm{~Hz}$ [6].

For computing the Lorentz detuning $\Delta f_{L}$, we first calculate the cavity deformation when the walls are subjected to radiation pressure, then we use Slater formulae:

$$
\frac{\Delta f_{L}}{f_{O}}=\frac{1}{4 W} \cdot \iiint_{\Delta V} P \cdot d V
$$


where $\Delta \mathrm{V}$ is the change of the resonator volume due to radiation pressure, $\mathrm{W}$ is the stored electromagnetic energy and $\mathrm{f}_{0}$ is the fundamental mode frequency. As the radiation pressure increases quadratically with the accelerating field (e.g., $\mathrm{P} \alpha \mathrm{E}_{\mathrm{acc}}{ }^{2}$ ) and the cavity wall deformations are small $(<1 \mu \mathrm{m})$, the Slater formula leads to the following relationship:

$$
\Delta f_{L}=-K_{L} E_{a c c}^{2}
$$

In the above expression, the Lorentz detuning factor $\mathrm{K}_{\mathrm{L}}$ is a parameter depending on cavity material, its shape and the boundary conditions. As consequence, an additional RF power is needed to operate the cavity at a given accelerating field if Lorentz detuning is not compensated. The ratio of the RF power $\mathrm{P}_{\mathrm{RF}}$ to that needed in the ideal case $\mathrm{P}_{\mathrm{RF} 0}$ (e.g., zero detuning) is given by the expression:

$$
\frac{P_{R F}}{P_{R F 0}}=1+\alpha \cdot\left(\frac{\Delta f(t)}{\Delta f_{B W}}\right)^{2}
$$

where $\alpha$ is a constant depending on the matching conditions: for TESLA/ILC machine $\alpha=0.25$ with particle beam, $\alpha=1$ without particle beam. Briefly, ILC is a superconducting $\mathrm{e}^{+} / \mathrm{e}^{-}$collider operating in pulsed mode with a pulse duration of $1.3 \mathrm{~ms}$ and a repetition rate of $5 \mathrm{~Hz}$ at $\mathrm{E}_{\mathrm{acc}}=33 \mathrm{MV} / \mathrm{m}$ for a centre of mass energy $\mathrm{E}_{\mathrm{cm}}=800 \mathrm{GeV}$. The measured detuning factor, in pulsed mode, for several TESLA cavities in the cryomodule is $\mathrm{K}_{\mathrm{L}} \cong 0.5-1 \mathrm{~Hz} /(\mathrm{MV} / \mathrm{m})^{2}$ leading to a maximum frequency shift $\Delta \mathrm{f}_{\mathrm{L}}=1090 \mathrm{~Hz}$ at $\mathrm{E}_{\mathrm{acc}}=33 \mathrm{MV} / \mathrm{m}$ and this figure is much larger than the cavity bandwidth $\Delta \mathrm{f}_{\mathrm{BW}}=425 \mathrm{~Hz}$. For such large detuning, the RF power is increased by a factor 2.64 so as to control RF field amplitude and phase, in comparison to non detuned cavity. It is therefore mandatory to compensate Lorentz detuning in order to save substantially power and increase by the way acceleration efficiency. A dynamic compensation of Lorentz detuning, using piezoelectric actuators as active elements for deforming the resonator was successfully applied (Fig. 4) to TESLA cavities [7].

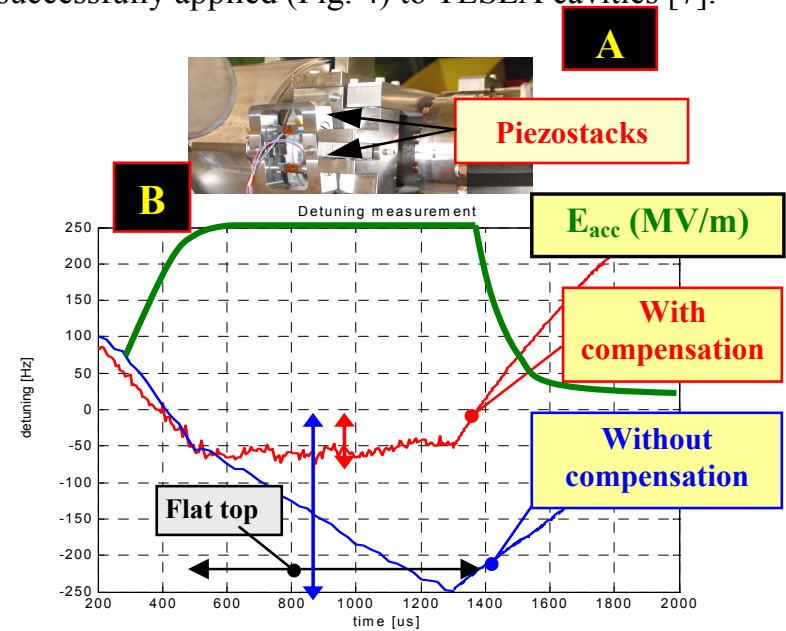

Fig. 4:A) Piezoelectric actuators attached to the tuning system mounted on a TESLA SRF cavity, B) Dynamic compensation of Lorentz detuning in TESLA cavity with piezoelectric actuators
From data of Fig. $4 \mathrm{~b}$ the measured Lorentz detuning in pulsed mode at a flat top gradient $\mathrm{E}_{\mathrm{acc}}=20 \mathrm{MV} / \mathrm{m}$, with and without compensation using piezostacks are respectively $\Delta \mathrm{f}_{\mathrm{L}} \sim-60 \mathrm{~Hz}$ and $\Delta \mathrm{f}_{\mathrm{L}} \sim-250 \mathrm{~Hz}$. Furthermore, during the flat top of $E_{a c c}$ (e.g., beam on phase) the detuning are respectively $\Delta \mathrm{f}_{\mathrm{L}} \sim-10 \mathrm{~Hz}$ (with compensation) and $\Delta \mathrm{f}_{\mathrm{L}} \sim-200 \mathrm{~Hz}$ (without compensation). This experiment clearly demonstrates the efficiency of piezoelectric actuators for dynamic compensation of Lorentz detuning in SRF cavities. However, due to the lack of data for the operating conditions of piezostacks in the cryomodule and accelerator environment (low pressure: $10^{-5} \mathrm{mbar}$, cryogenic temperatures, ionizing radiation), an experimental program aiming at full characterization of piezoelectric actuators at low temperature (i.e., $1.8 \mathrm{~K}-300 \mathrm{~K}$ ) was launched in the frame of the CARE project. In this paper, we will report the experimental results, we obtained recently.

\section{TEST APPARATUS}

\section{Piezoelectric Actuators}

A photograph of the three types of commercial piezoelectric actuators tested is shown in Fig. 5.

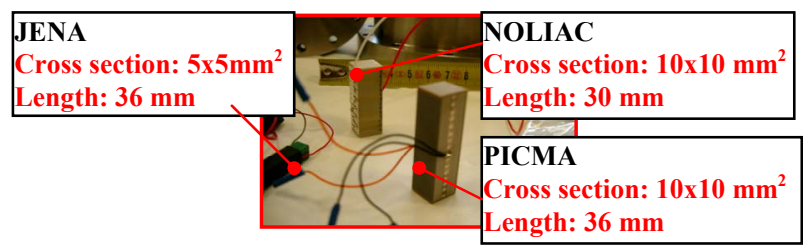

Fig. 5: Multilayer piezostacks base on Lead Zirconate Titanate material

The three types of low voltage multilayer piezostacks, which are based on Lead Zirconate Titanate material or PZT, were supplied by 3 different companies.

\section{Test Cell}

Three dedicated facilities were developed for the tests. The details of theses facilities as well as measurements method and experimental procedures were described previously [8-9]. The main elements of the test-cell are shown in Fig. 6.

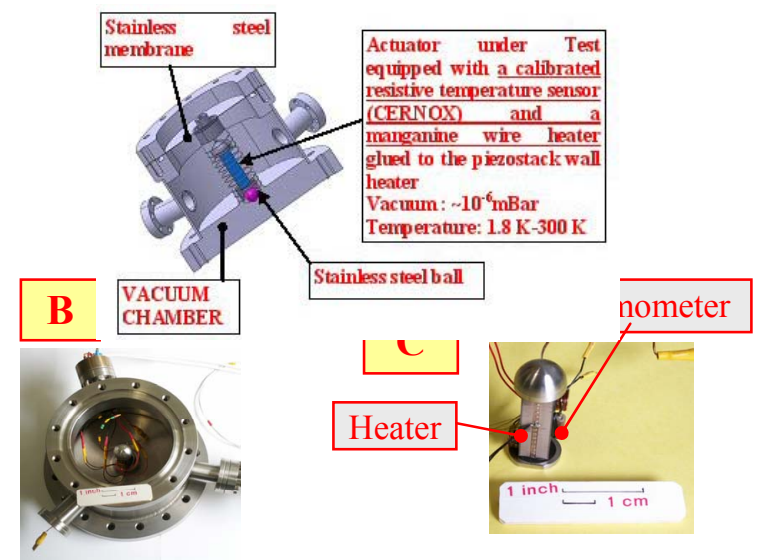

Fig. 6: A-Diagram of the test cell, B-Photograph of chamber with piezostacks during preparation, $C$-Close view to piezostacks equipped with heater and thermometer 
This test-cell consists mainly in a stainless steel cylindrical vacuum chamber with a removable thin end plate. The actuator is sandwiched between the lower rigid flange (thickness: $32.5 \mathrm{~mm}$ ) and the upper thin stainless steel sheet of $0.2 \mathrm{~mm}$ thickness. The system includes stainless steel ball with special fixture to avoid shear and torsion forces on the piezostacks, which is equipped with a heater, and a calibrated resistive CERNOX thermometer. When a voltage is applied to the piezoelectric actuator, it expands and deforms the upper thin sheet; resulting in a motion which is transmitted to the displacement sensor at room temperature via a stainless steel rod of $14 \mathrm{~mm}$ diameter.

\section{Feature of the Two First Facilities}

The two first facilities, which use the same test-cell, allow the full characterization of piezoelectric actuator under vacuum and at controlled low temperature in the range $1.8 \mathrm{~K}-300 \mathrm{~K}$. The following properties were investigated as function of temperature: a) calibration or displacement versus voltage characteristics, b) dielectric properties (capacitance: Cp, loss factor: $\operatorname{tg}(\delta)$, impedance), c) heating $\Delta \mathrm{T}$ due to dielectric losses when the actuator is subjected to a sinusoidal voltage, d) thermal properties (time constant: $\tau$, thermal resistance: $\mathrm{R}_{\mathrm{th}}$ and thermal capacitance: $\mathrm{C}_{\mathrm{th}}$ ), f) dynamic properties when the actuator is subjected to axial preloading force.

\section{RESULTS AND DISCUSSION}

\section{Actuators from Piezosystem JENA}

Several actuators of different production series from Piezosystem JENA were thoroughly investigated [8]. These actuators, which are already calibrated at room temperature by the supplier, were rejected owing to five main drawbacks and limitations: 1) the maximum displacement decreases strongly with temperature from $42 \mu \mathrm{m}$ at $\mathrm{T} \sim 300 \mathrm{~K}$ to less than $2 \mu \mathrm{m}$ at $2 \mathrm{~K}$ while $3 \mu \mathrm{m}$ are required, 2) they feature insufficient blocking force $\sim 1 \mathrm{kN}$ at $\mathrm{T}=300 \mathrm{~K}$ while $3 \mathrm{kN}$ are required, 3) the mechanical stiffness of $25 \mathrm{~N} / \mu \mathrm{m}$ is much lower than the $100 \mathrm{~N} / \mu \mathrm{m}$ required, 4) the fabrication is not reproducible from batch to batch leading to large variations in the piezostacks properties at low temperature, 5) the lifetime is very short $\left(\sim 5.10^{6}\right.$ cycles $)$ when operated dynamically at $2 \mathrm{~K}$ showing electromechanical breakdown and/or damages due to internal stresses.

\section{Actuators of PICMA and NOLIAC Type}

The piezostacks of PICMA type are a new generation of actuators fabricated by Physik Instruments and not calibrated. According to supplier and thank to new fabrication process, these actuators has a high durability and are suited for use at cryogenic temperatures. The non-calibrated actuators of NOLIAC type are multilayer piezostacks, also suited for use at low temperature. Therefore, we used actuator of JENA type and the displacement sensor as reference for the calibration of a PICMA and NOLIAC piezostacks.

\section{Calibration}

An example illustrating such calibration at $\mathrm{T}=300 \mathrm{~K}$ is presented in Fig. 7. The data show the well known hysteresis usually observed at $\mathrm{T}=300 \mathrm{~K}$ with a full range stroke of $40 \mu \mathrm{m}$ at the maximum voltage $\mathrm{V}_{\max }=120 \mathrm{~V}$ sustained by these actuators. It should be emphasised that this hysteresis is completely negligible at $\mathrm{T}<4.2 \mathrm{~K}$.

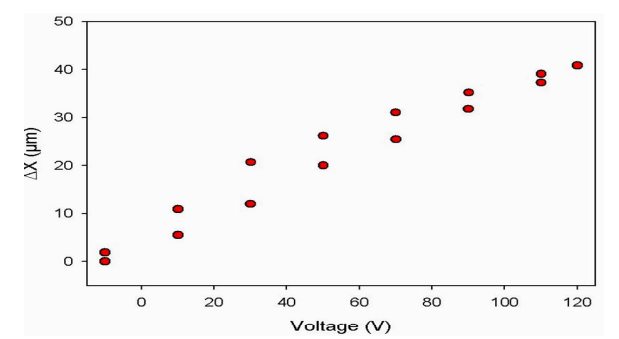

Fig.7: Calibration of actuator PICMA\#1at T=300 K

Systematic calibrations were performed for temperatures in the range $1.8 \mathrm{~K}-300 \mathrm{~K}$. The variations of full range displacement as function of temperature for PICMA and NOLIAC piezostacks are presented in Fig. 8. Obviously, the exact shape of the calibration curve depends on the microstructure of the ceramic material and the fabrication process. However, the data show the same non linear behaviour, namely a dramatic and monotonic decrease of full range displacement $\Delta \mathrm{X}$ with temperature $\mathrm{T}$, thus confirming the results previously reported [10-11]. Similarly to data obtained with Piezosystem JENA piezostacks [8], a strong decrease of the slope of $\Delta X$ vs. $T$ curve is observed with a vanishing value for $T<10 \mathrm{~K}$. This non linear behaviour was previously observed by other groups [1011]: depending on the piezostacks material $\Delta \mathrm{X}$ is decreased by a factor 5 to 20 when $\mathrm{T}$ is lowered from $300 \mathrm{~K}$ down to $\mathrm{T}=4 \mathrm{~K}$.

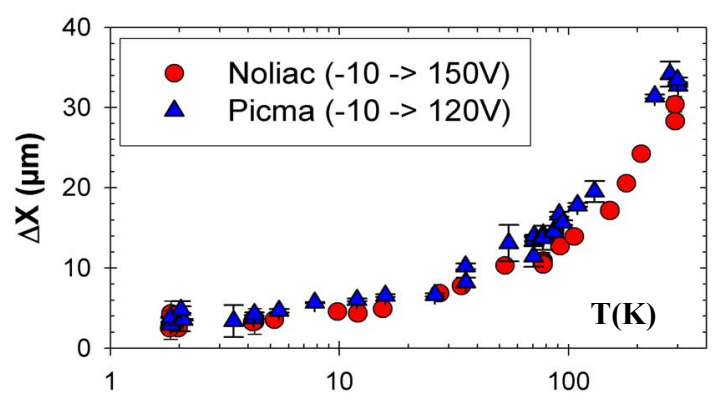

Fig. 8: Effect of temperature on full range displacement for actuators of PICMA and NOLIAC type

\section{Dielectric properties}

Dielectric properties (e.g., parallel capacitance: $\mathrm{Cp}$ and loss factor: $\operatorname{tg}(\delta))$ of the same actuators were measured at $100 \mathrm{~Hz}$ leading to the data displayed in Fig. 9. The experimental data show an exponential decrease of parallel capacitance with temperature. Further, it is obvious that $\Delta \mathrm{X}$ vs. $\mathrm{T}$ and $\mathrm{Cp}$ vs. $\mathrm{T}$ curves are homothetic, which suggests a tight correlation between parallel capacitance and full range displacement. 


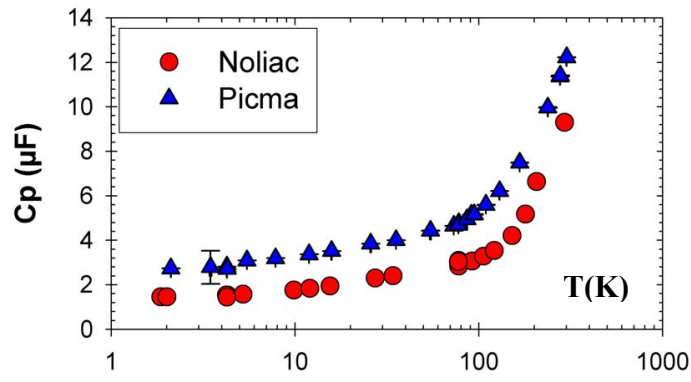

Fig.9: Effect of temperature on parallel capacitance for actuators of PICMA and NOLIAC type

This feature is clearly illustrated in Fig. 10 and confirms our observation with Jena piezostacks [8].

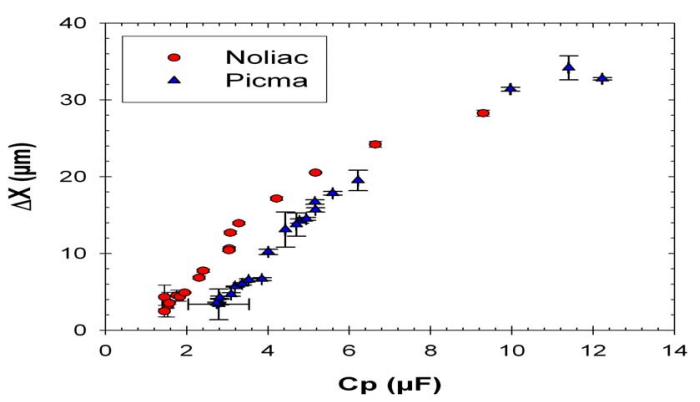

Fig. 10: Full range displacement versus capacitance

This correlation is very important, because it gives a simple mean for indirect calibration (e.g., $\Delta \mathrm{X}$ vs. $\mathrm{T}$ ) of a large number (i.e., 2000 actuators needed for XFEL, $\sim 36000$ actuators for ILC) of piezostacks. Indeed, capacitance measurements are easier and faster in comparison to true calibration (e.g., Displacement vs. actuator voltage for different T). Finally, the dielectric constant $\varepsilon_{\mathrm{r}}$ of the material vs. T, which is proportional to $\mathrm{Cp}$, is obviously homothetic to the curve of Fig.9. For PICMA piezostacks, $\varepsilon_{\mathrm{r}}$ decreases from 1454 at $\mathrm{T}=300 \mathrm{~K}$ to 328 at $\mathrm{T}=1.8 \mathrm{~K}$. The variations of loss factor as function of temperature are shown in Fig. 11. In contrast to capacitance data, the exact shapes of $\operatorname{tg}(\delta)$ vs. T curves of PICMA and NOLIAC actuators are quite different. However, they exhibit a broad peak respectively at $\mathrm{T} \approx 20 \mathrm{~K}$ for NOLIAC piezostacks and $\mathrm{T} \approx 90 \mathrm{~K}$ for PICMA actuators. This feature namely the peak in $\operatorname{tg}(\delta)$ vs. $\mathrm{T}$ curve was also observed for actuators from Piezosystem JENA and is common to PZT piezostacks. The exact shape seems tightly linked to the piezoelectric material (chemical composition, grain size) and the fabrication process which depends on the supplier.

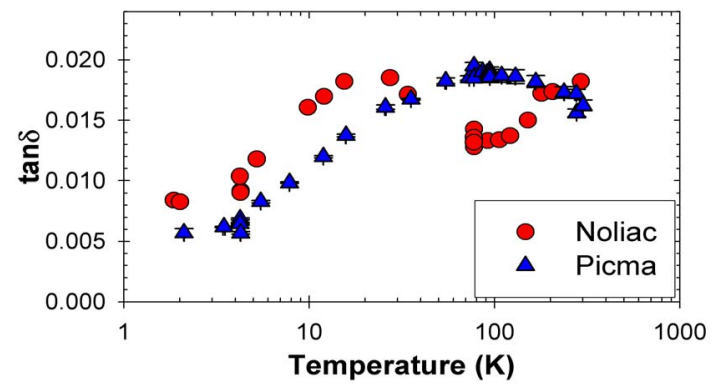

Fig. 11: Effect of temperature on loss factor for actuators of PICMA and NOLIAC type

\section{Dielectric heating}

Further, as the actuators will operate in dynamic condition for active tuning of SRF cavities, the effect of time varying voltage was investigated. The actuators are subjected to a sinusoidal voltage of amplitude $\mathrm{V}_{\text {mod }}$ and frequency $f_{\text {mod }}$ then the time variations of heating $\Delta T$ is recorded as illustrated in Fig. 12.

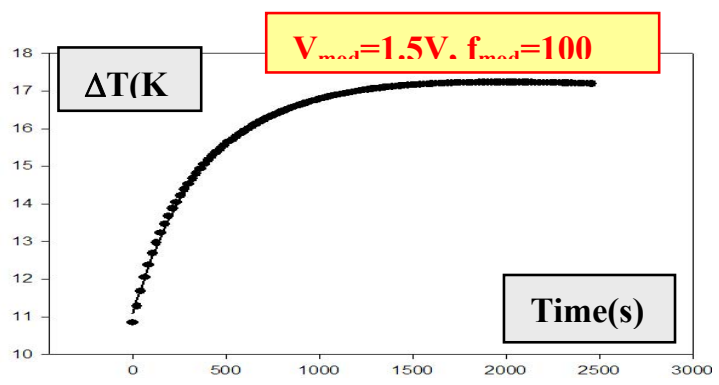

Fig. 12: Time variation of dielectric heating for PICMA actuator-Initial temperature $T_{0}=1.8 \mathrm{~K}$

Obviously, the time variations of heating are exponential $\left(\Delta \mathrm{T}(\mathrm{t})=\Delta \mathrm{T}_{\mathrm{ma}} \mathrm{x} .(1-\exp (-\mathrm{t} / \tau))\right.$. Obviously, the time constant is $\tau=\mathrm{R}_{\mathrm{th}} \cdot \mathrm{C}_{\mathrm{th}}$, where $\mathrm{R}_{\mathrm{th}}$ and $\mathrm{C}_{\text {th }}$ are respectively the interfacial thermal resistance and the thermal capacitance of the actuator. The capacitance is simply the product $\left(\mathrm{C}_{\mathrm{th}}=\mathrm{C}_{0} . \mathrm{m}\right)$ of specific heat $\mathrm{C}_{0}$ and mass of piezostacks. The fit to experimental data leads to the following values: $\tau=376 \mathrm{~s}, \mathrm{R}_{\mathrm{th}}=9.10^{5} \mathrm{mK} / \mathrm{mW}$ and $\mathrm{C}_{\mathrm{th}}=4.310^{-4} \mathrm{~J} / \mathrm{K}$. Note that $\mathrm{V}_{\text {mod }}$ corresponds to a displacement of $170 \mathrm{~nm}$ of the cavity wall (i.e. $\Delta \mathrm{f}=68$ $\mathrm{Hz}$ ) with total dielectric losses $\mathrm{P}_{\text {diel }}=20 \mu \mathrm{W}$ and heating $\Delta \mathrm{T}=17 \mathrm{~K}$. Moreover, the steady-state heating $\Delta \mathrm{T}$ vs. modulation voltage amplitude and frequency is shown in 3D plot of Fig. 13 for a PICMA actuator.

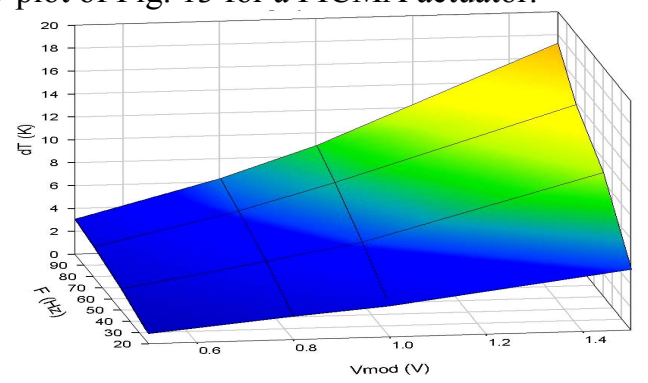

Fig. 13: Effect of amplitude and frequency on steady state dielectric heating.

The observed heating (i.e. $\Delta \mathrm{T}=\mathrm{R}_{\mathrm{th}} \cdot \mathrm{P}_{\text {diel }}$ ) is well described by the well-known expression of the total dielectric losses $\mathrm{P}_{\text {diel }}$ :

$$
P_{\text {diel }}=\pi \cdot C_{p} \cdot f_{\text {mod }} \cdot V_{\text {mod }}^{2} \cdot \sin (\delta)
$$

Note that the small departure from the quadratic dependence (5), observed at high modulation voltage amplitude and or frequency, is attributed to nonlinear effects resulting from $\mathrm{Cp}$ and $\sin (\delta)$ dependence on $\mathrm{T}$.

\section{PRELOADING EXPERIMENT}

The reliability and durability of piezostacks is mandatory: the requirements for ILC machine should be 
in excess of $3.210^{9}$ cycles in terms of actuators lifetime. Previous studies showed that the life time of piezostacks depends strongly on the applied preloading pressure $\mathrm{P}_{\text {Load }}$ with an optimum value $\mathrm{P}_{\text {Load }} \sim 100$ Bars at $\mathrm{T}=300 \mathrm{~K}$. In our best knowledge, few experimental data are for the operating conditions in the actual tuner are available. It is then necessary to perform an experimental investigation of electromechanical behaviour of actuators subjected to axial preload at cryogenic temperature. The main goals of this experiment are: 1) study the effect of a variable and controllable axial preload in the range $1 \mathrm{kN}-4 \mathrm{kN}$ on the electromechanical properties of the piezoelectric actuator (stroke, capacitance, loss factor), 2) development of a procedure for applying, adjusting and controlling the preload, 3) study of the behaviour of piezostacks as force sensor, 4) measurement of the mechanical stiffness of the actuator. A device dedicated to low temperature preloading experiment, as sketched in Fig. 14, was designed and constructed and successfully operated [12]. Briefly, a controlled vertical force is applied to the piezostacks via a high stiffness transmission rod by means of a rotating arm located at room temperature ( $\mathrm{T} \sim 300 \mathrm{~K})$ outside the cryostat. The force is simply adjusted by varying the load at the extremity of the rotating arm.

\section{Sensitivity to preloading at $\mathbf{T}=\mathbf{2 . 0 5 K}$}

The sensitivity of the actuator to preloading was measured at $\mathrm{T}=2.05$ for increasing and decreasing load. The variations of the relative capacitance $\Delta \mathrm{Cp}=\mathrm{Cp}-\mathrm{Cp}_{0}$, with reference to the capacitance $\mathrm{Cp}_{0}$ of the actuator when it is subjected to the arm preload $F_{0}=733 \mathrm{~N}$, as function of the preloading force $\mathrm{F}$ are shown in Fig. 15.

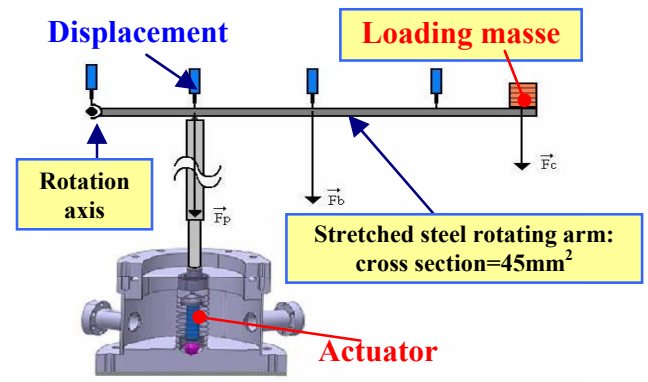

Fig. 14: Principle of preloading experiment

Non linear effects are observed at low preloading force when $\mathrm{F}$ is increased from zero to $\sim 1.3 \mathrm{kN}$ : they are due to dissipative and irreversible phenomena in the preloading device mechanism such as friction.

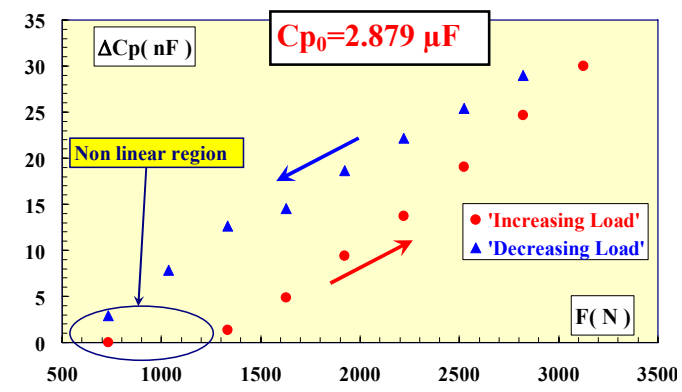

Fig. 15: Capacitance versus preload at $T=2.05 \mathrm{~K}$
Further, these data clearly show a large hysteresis for increasing and decreasing the preloading force. This hysteretic behaviour could be attributed to the intrinsic irreversibilities in the piezoelectric material itself.

\section{Effect of temperature on the sensitivity to preloading}

We investigate the effect of the actuator temperature on its sensitivity to preloading. Systematic measurements of $\Delta \mathrm{Cp}$ vs. $\mathrm{F}$ were performed for $\mathrm{T}$ in the range $1.7 \mathrm{~K}$ $300 \mathrm{~K}$. These results clearly show a linear dependence of the capacitance variation, at a given temperature, with the applied preload in the whole temperature range. Further, the non linear effects observed at $\mathrm{T}=2 \mathrm{~K}$ and low preloading force, were confirmed for all temperatures. Moreover the sensitivity to preloading, in the linear region, depends strongly on the temperature as illustrated in Fig. 16: each data point is simply the slope of the $\Delta \mathrm{Cp}$ vs. $\mathrm{F}$ curve at a given $\mathrm{T}$.

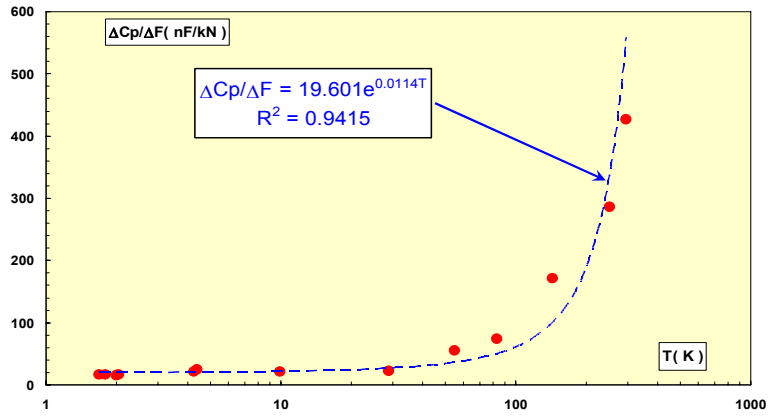

Fig. 16: Sensitivity to preloading versus temperature

More precisely, $\Delta \mathrm{Cp} / \Delta \mathrm{F}$ shows exponential temperature dependence: in the increasing preload mode, the slope $\Delta \mathrm{Cp} / \Delta \mathrm{F}$ increases with $\mathrm{T}$ from $16 \mathrm{nF} / \mathrm{kN}$ at $\mathrm{T}=2 \mathrm{~K}$ to $426 \mathrm{nF} / \mathrm{kN}$ at $\mathrm{T}=300 \mathrm{~K}$.

\section{Piezostacks as dynamic force sensor}

The behaviour of the piezostacks as dynamic force sensor was also studied by recording the transient response of the actuator when subjected to various negative and positive steep preloads variations $\Delta \mathrm{F}= \pm \mathrm{nx} 150 \mathrm{~N}$ at $\mathrm{T}=4.2 \mathrm{~K}$ as shown in Fig. 17.

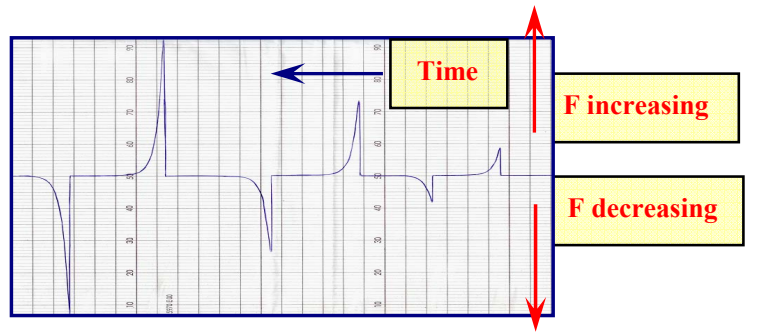

Fig. 17: Transient response of a PICMA actuator to various steep preload variation $\Delta F= \pm n x 150 N(n=1,2,3)$ at $T=4.2 \mathrm{~K}$.

The results call for the following remarks: 1) a steep voltage increase (capacitor charging) followed by an exponential decrease (capacitor discharging) is observed, 2) the peak actuator voltage $\Delta V_{p}$ is repeatable to within $3 \%, 3)$ the phenomenon is reversible and the response is linear, namely the peak voltage is proportional to preload variation $\left(\Delta \mathrm{V}_{\mathrm{p}} \propto \Delta \mathrm{F}\right)$ and change de sign with $\Delta \mathrm{F}$. 


\section{RADIATION TESTS WITH FAST NEUTRONS AT 4.2 K}

We investigated the effect of fast neutrons at $\mathrm{T}=4.2 \mathrm{~K}$ on piezoelectric actuator properties after an exposure to a dose in excess of $10^{14}$ neutrons $/ \mathrm{cm}^{2}$ in $\sim 20$ hours. Experimental details and discussion of the results are given elsewhere [13]. Three beam tests were performed. After cool down to $\mathrm{T}=4.2 \mathrm{~K}$, the dielectric properties are measured without beam. Then, the actuators are subjected to fast neutrons radiation and the parameters are on-line measured. The neutrons fluence distribution was off-line measured by means of an activation method of high purity nickel foils attached to the actuators. The final dielectric properties measured after neutrons exposure, are compared to the values before radiation as illustrated in Fig. 19-Fig. 20.

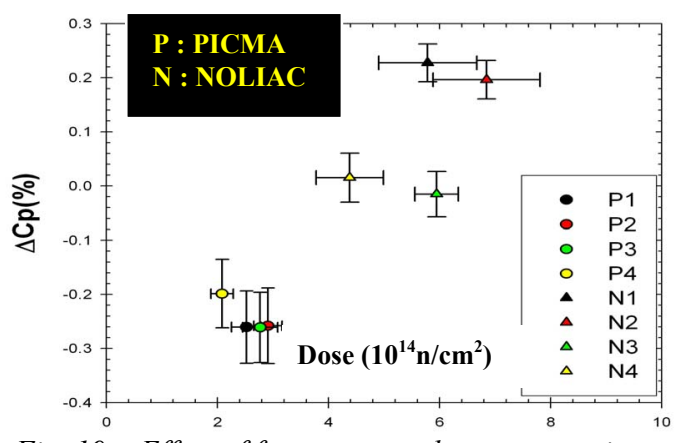

Fig. 19: Effect of fast neutrons dose on capacitance at $T=4.2 \mathrm{~K}$

To summarize, in the range of fast neutrons dose investigated namely $2.10^{14}-7.10^{14} \mathrm{n} / \mathrm{cm}^{2}$ the data lead to the following observations when neutrons fluence increases: 1) for PICMA actuators $\mathrm{Cp}$ decreases linearly by $0.25 \%, 2$ ) for NOILIAC actuators $\mathrm{Cp}$ increases linearly by $0.15 \%, 3$ ) for both actuators loss factor increases by 5 to $10 \%$.

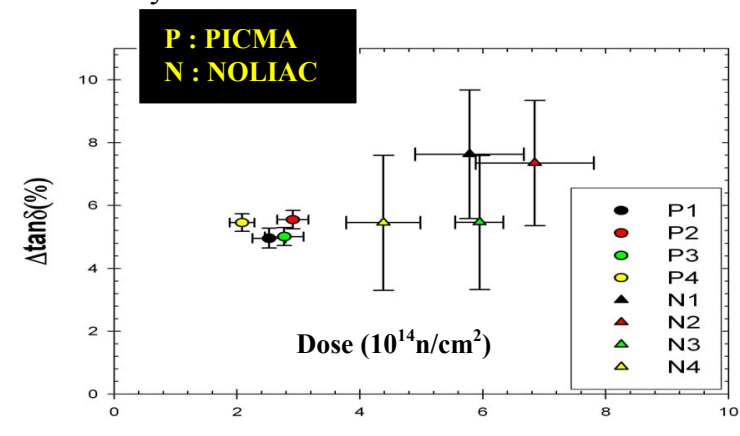

Fig. 20: Effect of fast neutrons dose on loss factor at $T=4.2 \mathrm{~K}$

\section{CONCLUSION}

Several low voltage multilayer piezostacks based on PZT material were fully characterized at cryogenic temperatures down to $1.8 \mathrm{~K}$. Piezostacks of PICMA and NOLIAC types fulfil all the requirements needed for active compensation of Lorentz detuning up to $1 \mathrm{kHz}$ frequency shift in SRF niobium TESLA cavities operating at $\mathrm{E}_{\mathrm{acc}}=33 \mathrm{MV} / \mathrm{m}$. The dynamic performances of these actuators allow operating ILC machine in pulsed mode with optimum transmission of RF power to electrons and positrons beams. The piezostacks could be also used as dynamic and static sensors for in situ measurements of force to which they are subjected during different stages of fast tuner operation in the cryomodule. Finally, as no major damage was observed during irradiations tests with fast neutrons at $\mathrm{T}=4.2 \mathrm{~K}$ for fluence up to a fluence $7.10^{14} \mathrm{n} / \mathrm{cm}^{2}$, PICMA and NOLIAC piezostacks are suited for use in cryogenic and neutrons radiation environment. The negligible observed performance after irradiations is probably due to aging effect.

\section{ACKNOWLEDGEMENTS}

We acknowledge the support of the European Community-Research Infrastructure Activity under the FP6 "Structuring the European Research Area" program (CARE, contract number RII3-CT-2003-506395)

\section{THE AUTHORS}

M. Fouaidy, G. Martinet, N. Hammoudi, F. Chatelet, A. Olivier are with IPNO, Université Paris-Sud 11, CNRS/IN2P3, 91406 Orsay, France.

\section{REFERENCES}

[1] C. Reece et al. 'Refining and maintaining the optimal performance of the CEBAF SRF systems', Proc. of PAC 2001,Chicago-USA, 1186-1188, 2001

[2] T.P. Wangler 'Design of a proton superconducting linac for a neutron spallation source', Proc. of $9^{\text {th }}$ workshop on RF superconductivity, 336, 1999.

[3] R. Brinkmann et al., 'TESLA XFEL, Technical Design Report Supplement', DESY 2002-167, 2002.

[4] A. Gamp, 'On the preference of cold RF technology for the international linear collider', TESLA Report 2005-23, 2005.

[5] J. L. Biarrotte et al., "704 MHz Superconducting Cavities for a High Intensity Proton Accelerator', $9^{\text {th }}$ Workshop on RF Superconductivity, Santa Fe, USA, Nov. 1999, p.384.

[6] H. Gassot, PhD thesis, Univ. Paris 11, Déc. 2001.

[7] M. Liepe, W.D Moeller, S. Simrock, DESY, Hamburg, "Dynamic Lorentz compensation with a fast piezoelectric tuner", Proc. PAC 2001.

[8] M. Fouaidy, N. Hammoudi, "characterization of piezoelectric actuators used for SRF cavities active

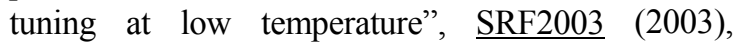
Lubeck-Travemunde, Germany

[9] M. Fouaidy et al. "Full characterization at low temperature of piezoelectric actuators used for SRF cavities active tuning", PAC05 (2005) Knoxville, Tennessee, USA, 728-730.

[10] K. G. Vandervoort et al., Rev. Sci. Instrum., Vol. 64, (1993), p. 896-899.

[11] M. Locatelli M. et al., Rev. Sci. Instrum, (1988) Vol. 59, p. 661.

[12] M. Fouaidy et al. "Electromechanical characterization of piezoelectric actuators subjected to a variable preloading force at cryogenic temperature ", CARE-Note-2006-007-SRF 